\title{
Bevacizumab intravítreo como monoterapia de la retinopatía del prematuro en 12 pacientes
}

\author{
Intravitreal bevacizumab as single drug therapy for retinopathy of prematurity \\ in 12 patients
}

\author{
Dr. Aldo Bancalari M., ${ }^{a}$ Dr. Ricardo Schade Y., ${ }^{b}$ Dr. Rubén Peña Z., ${ }^{c}$ Dr. Nicolás Pavez P. ${ }^{d}$
}

\begin{abstract}
RESUMEN
Comunicamos los resultados oftalmológicos en una serie de niños tratados con bevacizumab intravítreo con retinopatía del prematuro (RDP) en etapa umbral.

Se evaluaron doce recién nacidos pretérmino de muy bajo peso (RNMBP) que recibieron bevacizumab intravítreo como monoterapia en el tratamiento de la retinopatía del prematuro en etapa umbral y que presentaban contraindicación para la terapia estándar con fotocoagulación con láser. Se realizaron controles oftalmológicos y se evaluó la respuesta al tratamiento, reintervenciones y complicaciones.

La edad gestacional de estos pacientes fue $26,3 \pm 1,8$ semanas y el peso de nacimiento de $845 \pm 153 \mathrm{~g}$. Hubo buena respuesta en ocho casos, mientras que cuatro pacientes requirieron reintervención con fotocoagulación con láser. No se detectaron complicaciones inmediatas y no hubo pacientes fallecidos.

Palabras clave: retinopatía de la prematuridad, inyecciones intravítreas, bevacizumab.
\end{abstract}

http:/ /dx.doi.org/10.5546/aap.2014.160

\section{INTRODUCCIÓN}

La retinopatía del prematuro (RDP) es un desorden proliferativo del desarrollo vascular de la retina. Constituye la principal causa de ceguera en niños en países desarrollados y en vías de desarrollo. ${ }^{1}$ En un estudio efectuado en Chile, se demostró que la RDP fue la principal causa de ceguera infantil. ${ }^{2}$ La incidencia de esta enfermedad aumenta con menor peso de nacimiento y edad gestacional. ${ }^{3}$

a. Unidad de Neonatología, Servicio de Pediatría.

b. Servicio de Oftalmología.

Hospital Guillermo Grant Benavente, Concepción, Chile.

c. Residente de Pediatría.

d. Residente de Medicina Interna.

Facultad de Medicina, Universidad de Concepción.

Correspondencia:

Dr. Aldo Bancalari Molina: aldobancalari@gmail.com

Conflicto de intereses: Ninguno que declarar.

Recibido: 29-5-2013

Aceptado: 7-10-2013
En la patogénesis de la RDP, se describe una primera fase caracterizada por un ambiente con elevadas concentraciones de oxígeno en la arteria retinal y bajos niveles circulantes de factor de crecimiento endotelial vascular (VEGF), seguida por una segunda fase de proliferación vascular inducida por hipoxia, con elevados niveles de VEGF. ${ }^{4}$ El uso de medicamentos inhibidores del VEGF, como el anticuerpo monoclonal bevacizumab, ha mostrado resultados promisorios en el tratamiento de la retinopatía en etapa umbral. ${ }^{5}$

El bevacizumab ha sido aprobado por la Administración de Medicamentos y Alimentos (Food and Drug Administration, FDA) para tratar algunos tipos de cáncer; pero aún no ha sido aprobado como tratamiento para la RDP. ${ }^{6}$ Por su mecanismo de acción, se ha usado en la RDP en etapa umbral como monoterapia o coadyuvante a la terapia con fotocoagulación con láser (FCL).5,6

Cabe mencionar que la terapia estándar para el tratamiento de la RDP es la FCL, la cual puede presentar complicaciones, como quemadura de la córnea o iris, hemorragia retinal e hifema, ${ }^{7}$ lo que ha motivado la búsqueda de nuevas alternativas terapéuticas en casos seleccionados.

\section{OBJETIVO}

Presentar los resultados oftalmológicos con la administración de bevacizumab intravítreo en una serie de recién nacidos prematuros con retinopatía en etapa umbral.

\section{MATERIAL Y MÉTODO}

Entre enero de 2008 y diciembre de 2012, se analizaron las historias clínicas de recién nacidos pretérmino de muy bajo peso (RNMBP) en los que se administró bevacizumab (Avastin ${ }^{\circledR}$ ) por presentar RDP en etapa umbral y la imposibilidad de utilizar la terapia estándar con FCL en primera instancia por las siguientes situaciones: dilatación incompleta por rubeosis, enfermedad en zona posterior y contraindicación de anestesia general por inestabilidad del prematuro. El uso de 
bevacizumab en nuestro centro se encuentra aprobado por el Comité de Ética en los casos seleccionados de RDP antes señalados. En todos los neonatos, se obtuvo consentimiento informado escrito de los padres antes del tratamiento y se explicó la indicación, la forma de administración y las posibles complicaciones del fármaco.

El examen oftalmológico se inició a las 4 semanas de nacimiento, por oftalmología indirecta, y se realizó un control semanal. La clasificación de RDP se efectuó de acuerdo con la Clasificación Internacional de Retinopatía. ${ }^{8}$ Los candidatos para el tratamiento fueron aquellos pacientes que alcanzaron retinopatía en etapa 3 plus (+) y en etapa 2 plus cuando existió compromiso en zona I o II posterior.

La sedación del recién nacido (RN) se realizó con fentanilo y el globo ocular se anestesió en forma tópica con proparacaína. Se inyectó bevacizumab en una dosis de $0,625 \mathrm{mg}$ a través de pars plana a $2 \mathrm{~mm}$ del limbo (Figura 1). Luego de la intervención, se indicó profilaxis antibiótica con colirio de tobramicina y dexametasona cada 3 horas por tres días. Posteriormente, se realizaron controles oftalmológicos seriados, al día siguiente y luego cada semana hasta los 3 meses y en forma mensual hasta alcanzar la edad corregida de un año. El medicamento fue administrado en la Unidad de Neonatología y el seguimiento general y neurológico se realizó según cronograma nacional de atención al recién nacido.

En cada paciente, se evaluó la respuesta al tratamiento y las complicaciones con su uso, como hifema, endolftalmitis o desprendimiento de retina, así como también la necesidad de

FIGURA 1. Administración intravítrea de bevacizumab en la Unidad de Neonatología

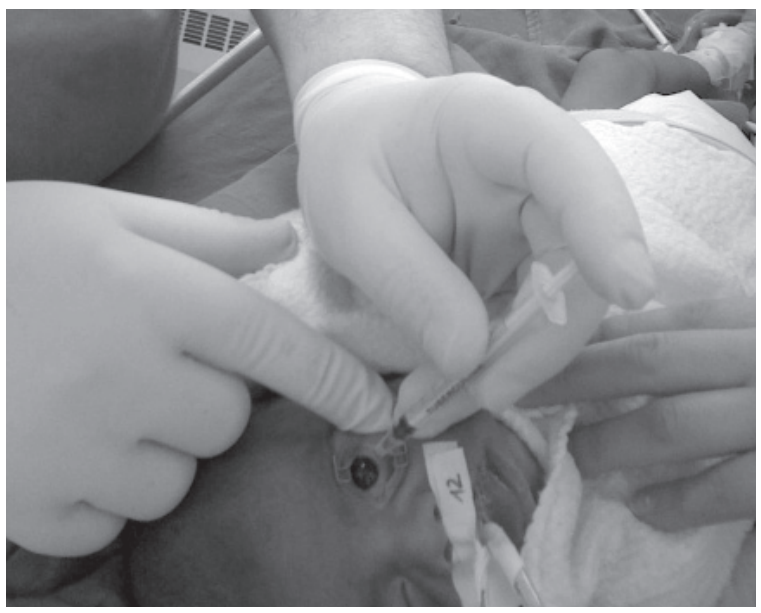

reintervención. Se consideraron candidatos a reintervención mediante FCL aquellos pacientes que en el seguimiento progresaron o se mantuvieron en etapa $2+$ posterior o etapa 3 , luego de haber administrado el bevacizumab intravítreo.

\section{RESULTADOS}

Se trataron 12 RNMBP con una edad gestacional y peso de nacimiento promedio \pm DE de 26,3 $\pm 1,8$ semanas y $845 \pm 153 \mathrm{~g}$, respectivamente. La edad posnatal promedio $\pm \mathrm{DE}$ al momento de la administración del bevacizumab fue de 7,8 $\pm 2,1$ semanas. La distribución por género fue semejante. Los datos demográficos individualizados de cada neonato se presentan en la Tabla 1. La administración más precoz de bevacizumab fue a las 4 semanas de vida, que corresponde al RN de mayor peso (1120 g), y la más tardía, a las 10 semanas en el RN de $680 \mathrm{~g}$ al nacer (paciente 3).

Al momento de administrar el bevacizumab, 8 de los 12 pacientes presentaban RDP en etapa $3+$ (Tabla 1) y los 4 restantes se encontraban en etapa $2+$. Uno de los neonatos evaluados presentó hemorragia retinal (paciente 1). La zona más comprometida fue la zona II en 8 de los 12 casos.

De los neonatos que recibieron bevacizumab, en 4 de ellos se necesitó posteriormente efectuar tratamiento con FCL (Tabla 1). Las reintervenciones fueron necesarias porque la RDP progresó a etapa 3 , habiéndose documentado previamente una fase de regresión a etapa 1. En los 8 pacientes restantes, la evolución fue satisfactoria y no requirieron nueva intervención.

Al realizar los controles oftalmológicos seriados, no se encontraron complicaciones, como hifema, endoftalmitis, desprendimiento de retina o catarata. No obstante, en el control al año del paciente 10 (Tabla 1), se observó tortuosidad vascular con leve tracción macular en el ojo derecho. No hubo mortalidad en los niños estudiados.

\section{DISCUSIÓN}

En nuestra serie, el tratamiento con bevacizumab se inició en 2008 con la finalidad de tratar pacientes con diagnóstico de RDP en etapa umbral, en los que no fue posible realizar fotocoagulación con láser en el momento en que estaba indicada. Cabe hacer notar que el tratamiento estándar de la RDP en etapa umbral es la FCL; sin embargo, los agentes anti-VEGF, como el bevacizumab, han sido utilizados en casos seleccionados con resultados prometedores. ${ }^{9}$ 
162 / Arch Argent Pediatr 2014;112(2):160-168 / Comunicaciones breves

TABLA 1. Características demográficas y compromiso de los pacientes con retinopatía del prematuro tratados con bevacizumab

\begin{tabular}{|c|c|c|c|c|c|c|c|c|}
\hline Paciente & $\begin{array}{c}\mathrm{EG}^{*} \\
(\text { sem.) }\end{array}$ & Sexo & $\begin{array}{c}\text { Peso } \\
(\mathrm{g})\end{array}$ & Etapa & Zona & $\begin{array}{c}\text { EG en el momento } \\
\text { del tratamiento (sem.) }\end{array}$ & Tratamiento & Reintervención \\
\hline 1 & 26 & $\mathrm{~F}$ & 850 & $3+$ & 2 & 36 & Bevacizumab & \\
\hline 2 & 27 & $\mathrm{~F}$ & 900 & $3+$ & 2 & 35 & Bevacizumab & Láser \\
\hline 3 & 26 & M & 680 & $3+$ & 3 & 36 & Bevacizumab & \\
\hline 4 & 25 & $\mathrm{~F}$ & 1120 & $2+$ & 2 & 29 & Bevacizumab & \\
\hline 5 & 26 & $\mathrm{~F}$ & 900 & $2+$ & 2 & 34 & Bevacizumab & Láser \\
\hline 6 & 25 & M & 720 & $3+$ & 2 & 35 & Bevacizumab & \\
\hline 7 & 31 & $\mathrm{~F}$ & 1020 & $2+$ & 1 & 36 & Bevacizumab & \\
\hline 8 & 24 & $\mathrm{~F}$ & 610 & $3+$ & 2 & 32 & Bevacizumab & Láser \\
\hline 9 & 26 & M & 980 & $2+$ & 1 & 35 & Bevacizumab & \\
\hline 10 & 27 & M & 890 & $3+$ & 2 & 32 & Bevacizumab & Láser \\
\hline 11 & 25 & M & 780 & $3+$ & 2 & 36 & Bevacizumab & \\
\hline 12 & 28 & M & 680 & $3+$ & 2 & 35 & Bevacizumab & \\
\hline
\end{tabular}

* EG: edad gestacional; sem.: semanas; g: gramos.

Desde el punto de vista fisiopatológico, la retinopatía del prematuro se caracteriza por proliferación vascular mediada por niveles altos de VEGF, por lo que, al utilizar un inhibidor de esta molécula, se pretende efectuar un tratamiento dirigido hacia el mecanismo de producción de la enfermedad..$^{10}$ En paralelo, la inyección intravítrea con bevacizumab es una intervención menos agresiva sobre la retina, que evita la destrucción de retina periférica, que suele ocurrir con la utilización de láser. ${ }^{11}$

Recientemente, se publicó el primer estudio prospectivo multicéntrico aleatorizado en 150 pacientes, que comparó la eficacia del bevacizumab versus la fotocoagulación con láser en la RDP etapa $3+.{ }^{5}$ Este ensayo clínico mostró como resultado un $4 \%$ de recurrencia con bevacizumab como monoterapia y un $22 \%$ en el grupo tratado con FCL, diferencia estadísticamente significativa. ${ }^{5}$ La dosis de bevacizumab en este estudio fue la misma utilizada en nuestra experiencia $(0,625 \mathrm{mg})$.

Se han comunicado escasas complicaciones con el uso de bevacizumab. Algunas de ellas corresponden a hifema, endoftalmitis, desprendimiento de retina o catarata. ${ }^{12}$ En nuestros pacientes, no observamos estas complicaciones.

Dado el rol angiogénico del VEGF necesario para la formación de nuevas estructuras, entre las que se encuentran el sistema nervioso central y el desarrollo alveolar pulmonar, ${ }^{13}$ es importante realizar nuevos estudios con seguimiento de estos pacientes para fundamentar el uso del bevacizumab intravítreo en la RDP.

A pesar de no disponer de suficiente información como para recomendar su uso, el tratamiento de la RDP en etapa umbral con bevacizumab intravítreo podría presentar ventajas respecto al tratamiento con fotocoagulación con láser. ${ }^{5,14}$ Esta terapia sería más específica de acuerdo con la patogénesis de la enfermedad, con posible menor pérdida de campo visual y con la facilidad de usar solo anestesia local. ${ }^{15} \mathrm{No}$ obstante, es necesario efectuar un seguimiento a mediano y largo plazo en los pacientes tratados con bevacizumab para evaluar posibles efectos locales y sistémicos.

\section{CONCLUSIONES}

En nuestra casuística, ninguno de los pacientes tratados con bevacizumab intravítreo presentó complicaciones oculares inmediatas. Se observó una respuesta satisfactoria a la monoterapia en 8 de los 12 niños.

\section{BIBLIOGRAFÍA}

1. GilbertC. Retinopathy of prematurity: a global perspective of the epidemics, population of babies at risk and implications for control. Early Hum Dev 2008;84:77-82.

2. GilbertC,Cánovas R, Kocksch R, Foster A. Ceguera infantil en Chile: estudio de escuelas de ciegos. Arch Chil Oftalmol 1993;50:49-53.

3. Bancalari A, González R, VásquezC,Pradenas I. Retinopatía del prematuro: incidencia y factores asociados. Rev Chil Pediatr 2000;71:114-21.

4. Chen J, Smith LE. Retinopathy of prematurity. Angiogenesis 2007;10:133-40. 
5. Mintz-Hittner HA, Kennedy KA, Chuang AZ. Efficacy of intravitreal bevacizumab for stage 3+ retinopathy of prematurity. N Engl J Med 2011;364:603-15.

6. FDA. Avastin Approval History. U.S. Food and Drug Administration. [Acceso: 22 de mayo de 2013]. Disponible en: http://www.accessdata.fda.gov/drugsatfda_docs / label/2009/125085s0169lbl.pdf.

7. ClarkD,MandalK. Treatment of retinopathy of prematurity. Early Human Develop 2008;84:95-9.

8. International Committee for the Classification of Retinopathy of Prematurity. The InternationalClassification of Retinopathy of Prematurity revisited. Arch Ophthalmol 2006;124:1669-70

9. Micieli JA, Surkont M, Smith AF. A systematic analysis of the off-label use of bevacizumab for severe retinopathy of prematurity. Am J Ophthalmol 2009;148:536-43.

10. Travassos A, Teixeira S, Ferreira P, Regadas I, et al. Intravitreal bevacizumab in aggressive posterior retinopathy of prematurity. Ophthalmic Surg Lasers Imaging 2007;38:233-7.
11. The Early Treatment of Retinopathy Cooperative Group. Final visual acuity results in the Early Treatment for Retinopathy of Prematurity Study. Arch Ophthamol 2010;128(6):663-71.

12. Jamrozy-Witkowska A, Kowalska K, Jankowska-Lech I, Terelak-Borys B, et al. Complications of intravitreal injections--own experience. Klin Oczna 2011;113:127-31.

13. Thébaud B, AbmanS. Bronchopulmonary dysplasia. Where have all the vessels gone? Roles of angiogenic growth factors in chronic lung disease. Am J Respir Crit Care Med 2007;175:978-85.

14. Darlow BA, Gilbert C, Quinn GE, Azad R, et al. Promise and potencial pitfalls of anti VEGF drugs in Retinopathy of Prematurity. Br J Ophthalmol 2009;93(7):986.

15. Castellanos MA, Schwartz S, Leal R, Chan RV, et al. Pain assessment in premature infants treated with intravitreal antiangiogenic therapy for retinopathy of prematurity under topical anesthesia. Graefes Arch Clin Exp Ophthalmol 2013;251:491-4

\title{
Infección grave por Staphylococcus aureus en tres unidades de cuidados intensivos pediátricos. Análisis de los casos de neumonía necrotizante
}

\author{
Severe Staphylococcus aureus infection in three pediatric intensive care units. \\ Analysis of cases of necrotizing pneumonia
}

\author{
Dr. Pedro Taffarel ${ }^{a}$, Dr. Germán Bonetto ${ }^{b}$, Dr. Matías Penazzia,c, Dr. Facundo Jorro Barón ${ }^{a}$, \\ Dra. Silvia Saenz $z^{b}$ Dra. Macarena Uranga ${ }^{d}$, Dr. Eduardo Maric, Bioq. Rosana Peredae y Dr. Gustavo Debaisia
}

\section{RESUMEN}

El Staphylococcus aureus afecta frecuentemente al ser humano. Dentro delas manifestaciones clínicas, la neumonía necrotizante se asocia a una alta mortalidad. Nuestro objetivo es describir la evolución de las infecciones graves por Staphylococcus aureus en tres unidades de terapia intensiva pediátricas y analizar los casos

a. Servicio de Terapia Intensiva del Hospital General de Niños Pedro de Elizalde.

b. Servicio de Terapia Intensiva del Hospital de Niños de la Santísima Trinidad, Córdoba.

c. Servicio de Terapia Intensiva del Hospital del Niño de San Justo.

d. Servicio de Infectología del Hospital del Niño de San Justo.

e. Servicio de Microbiología del Hospital General de Niños Pedro de Elizalde.

Correspondencia:

Pedro Taffarel: Pedrotaffarel@hotmail.com

Conflicto de intereses: Ninguno que declarar.

Recibido: 24-6-2013.

Aceptado: 22-11-2013. de neumonía necrotizante en el período del 01-2011 al 03-2013. Se analizaron 43 pacientes; $76,7 \%$ presentaron infección adquirida en la comunidad, y en 31 fue por Staphylococcus aureus resistente a la meticilina adquirido en la comunidad. El principal motivo de ingreso fue la claudicación respiratoria. Se documentó bacteriemia en el 55,8\% de los casos. El 86\% de los ingresos requirieron asistencia respiratoria mecánica y 27 pacientes desarrollaron shock séptico. La estadía en la unidad de terapia intensiva fue de 13 (5-25) días, y la mortalidad, del $14 \%$. La neumonía necrotizante estuvo presente en el $51 \%$ de los casos.

Conclusión. Se identificó una alta proporción de infección adquirida en la comunidad. La neumonía necrotizante se asoció con una peor evolución.

Palabras clave: Staphylococcus aureus, adquirido en la comunidad, neumonía necrotizante.

http:/ /dx.doi.org/10.5546/aap.2014.163

\section{INTRODUCCIÓN}

El Staphylococcus aureus (SA) es uno de los patógenos más importante que afecta al ser humano. Produce un amplio espectro de infecciones, desde leves, que involucran la piel, hasta graves, como bacteriemia y sepsis. ${ }^{1}$ 
En nuestro país, los estudios de Paganini reflejan la tendencia creciente de las infecciones por Staphylococcus aureus resistente a la meticilina (SAMR) adquiridas en la comunidad (SAMRAC). ${ }^{2-4}$

Dentro de las manifestaciones clínicas de la infección por SA, la más temida es la neumonía necrotizante (NMN-N), síndrome caracterizado por insuficiencia respiratoria y shock séptico de rápida evolución, en el contexto de fiebre, hemoptisis, leucopenia, consolidación multifocal, lesiones cavitarias pulmonares y derrame pleural, ${ }^{5}$ asociado a una alta mortalidad (56-63\%). ${ }^{6}$

Dentro de los factores de virulencia del SA, se encuentra la producción de una toxina llamada leucocidina de Panton Valentine (LPV). ${ }^{7}$

\section{OBJETIVO}

Describir las características clínicas y evolutivas de niños con infecciones graves por SA en tres unidades de terapia intensiva.

\section{MÉTODOS}

Estudio retrospectivo (del 1/1/2011 al $29 / 2 / 2012$ ) y prospectivo (del 1/3/2012 al $31 / 3 / 2013$ ), aprobado por el Comité de Ética. Se registraron todos los pacientes que ingresaron con infección grave por SA a los Servicios de Terapia Intensiva Pediátrica de los Hospitales Pedro de Elizalde (HNPE), Del Niño de San Justo (HNSJ) y Santísima Trinidad (HNST), sin incluir a los reingresos por complicaciones alejadas de dicha infección. La revisión de las historias clínicas, como el seguimiento de los pacientes, fue llevada a cabo por alguno de los tres primeros autores, $y$ las dudas se resolvieron por consenso.

Para realizar el hemocultivo, se utilizó el sistema Bact-alert, y la sensibilidad antibiótica se estudió según normas del Instituto de Estándares Clínicos y de Laboratorio (CLSI).

Se registró la edad, peso, puntaje PIM2 (índice de mortalidad pediátrica 2) y presencia de enfermedades previas. Se consideró foco primario aquel al que se atribuyó el inicio del proceso infeccioso, objetivado por clínica y/o microbiología. El motivo de ingreso a la Unidad de Terapia Intensiva Pediátrica (UTIP) fue clasificado en claudicación respiratoria (insuficiencia respiratoria y/o mala mecánica ventilatoria), falla hemodinámica o posquirúrgico.

Respecto al microorganismo SA, se registraron las variables infección nosocomial o adquirida en la comunidad, ${ }^{8}$ SAMR o Staphylococcus aureus meticilino sensible (SAMS), sitio donde se rescató el microorganismo, antibioticoterapia previa, antibiograma, retraso del tratamiento adecuado según antibiograma (expresado en horas) y localización metastásica, definida como infección no contigua al foco primario. ${ }^{9}$

En cuanto a la evolución en las UTIP, se registraron las variables asistencia respiratoria mecánica (ARM) y días libres de esta a los 28 días del ingreso, síndrome de dificultad respiratoria aguda (SDRA), ${ }^{10}$ ventilación de alta frecuencia oscilatoria (VAFO), shock séptico, compromiso hematológico, hepático y/o renal, ${ }^{11}$ necesidad de adrenalina $>0,25$ gamas $/ \mathrm{kg} / \mathrm{min}$ como expresión de bajo gasto cardíaco, puntaje de PELOD (Pediatric Logistic Organ Dysfunction). También se analizó la presencia de escape aéreo (evidencia radiológica de enfisema intersticial, neumotórax, neumomediastino y/o enfisema subcutáneo), de enfermedad pulmonar crónica (requerimiento de $\mathrm{O}_{2}$ suplementario luego de los 30 días del ingreso a ARM), como así también la necesidad de tratamiento quirúrgico y el tipo de cirugía.

Finalmente, se analizaron los casos de neumonía necrotizante (NMN-N), sus características clínicas y evolutivas y su asociación como factor predictor de peor evolución, la duración de la estadía en la UTIP y la mortalidad.

\section{Análisis estadístico}

Se utilizó el programa STATA 9.0; los valores fueron expresados en medias y desvíos estándar o medianas y rango intercuartilo, y analizados con el test-T de Student y la prueba de suma de rangos de Wilcoxon, respectivamente. Se utilizó la estadística de Chi cuadrado para comparar las variables dicotómicas. Se consideró significativo un valor $\mathrm{p}<0,05$.

La potencia de la muestra para el análisis de los casos de NMN-N se calculó retrospectivamente.

\section{RESULTADOS}

Durante el período analizado, ingresaron a las UTIP 50 pacientes con diagnóstico de infección grave por SA o por complicación de esta; siete fueron excluidos por considerarse reingresos. Se analizaron 43 pacientes (Tabla 1). El 76,7\% (33) de los pacientes presentaron infección adquirida en la comunidad; en 31 fue por SAMR-AC. De estos, un solo paciente padecía una enfermedad crónica concomitante. Diez pacientes manifestaron infección de origen hospitalario (Tabla 2); este grupo albergó 6 pacientes con enfermedades previas. El principal motivo de admisión a 
TABla 1. Principales características de los 43 pacientes

\begin{tabular}{|c|c|}
\hline Variable & $N(\%)-X \pm D E-M e / R I Q$ \\
\hline Edad (meses) & $22 / 6-72$ \\
\hline Género masculino & $26(60,5 \%)$ \\
\hline Peso corporal (kg) & $18,7 \pm 15,1$ \\
\hline PIM2 (\%) & $7,6 \pm 11,7$ \\
\hline \multicolumn{2}{|l|}{ Categorías diagnósticas/foco primario } \\
\hline - Pulmonar & $22(51 \%)$ \\
\hline - TCS-Muscular & $13(32,5 \%)$ \\
\hline - Osteoarticular & $4(7 \%)$ \\
\hline - NAR & $2(4,7 \%)$ \\
\hline - Meníngeo & $1(2,3 \%)$ \\
\hline$-\quad \mathrm{CVC}$ & $1(2,3 \%)$ \\
\hline - Enfermedad crónica subyacente & $7(16,3 \%)$ \\
\hline \multicolumn{2}{|l|}{ Categorías diagnósticas/motivo de ingreso } \\
\hline - Falla respiratoria & $22(51,2 \%)$ \\
\hline - Falla hemodinámica & $16(37,2 \%)$ \\
\hline - Posquirúrgico & $5(11,6 \%)$ \\
\hline
\end{tabular}

Variables relacionadas con Staphylococcus aureus

Infección adquirida en la comunidad $\quad 33(76,7 \%)$

$\begin{array}{ll}\text { SAMR } & 35(81,4 \%)\end{array}$

Antibiograma/resistencia

1. Vancomicina

2. Clindamicina

3. Rifampicina

$1(2,4 \%)$

4. Trimetoprima-sulfametoxazol

$1(2,4 \%)$

5. Gentamicina

$1(2,4 \%)$

Rescate bacteriológico
1. Hemocultivo
2. Líquido pleural
3. Aspirado traqueal
4. TCS-muscular
5. CVC
6. Líquido articular
7. Más de un sitio
7. Más de un sitio
8. Otros

$3(7 \%)$

$3(7 \%)$

$3(7 \%)$

$2(5 \%)$

$9(20 \%)$

$3(7 \%)$

$13(30 \%)$

Antibioticoterapia previa

$10(23 \%)$

\section{Variables relacionadas con la evolución en las UTIP}

Días de ARM

Días libres de ARM a los 28 días del ingreso

$17 / 4-24$

SDRA

VAFO

Síndrome escape aéreo 


$\begin{array}{lc}\text { Neumonía necrotizante } & 22(51 \%) \\ \text { Shock séptico } & 27(63 \%) \\ \text { Compromiso de órganos } & \\ \quad-\quad \text { Hematológico } & 16(37,2 \%) \\ -\quad \text { Renal } & 16(37,2 \%) \\ \quad-\text { Hepático } & 8(18,6 \%) \\ \quad \text { - PELOD } & 18,64+28,3 \\ \text { Adrenalina > } 0,25 \text { gamas } / \mathrm{kg} / \mathrm{min} & 20(46,5 \%) \\ \text { Estadía en la UTIP } & 13 / 5-25 \\ \text { Mortalidad } & 6(14 \%)\end{array}$

$\mathrm{N}(\%)$ : número (\%); $\mathrm{X} \pm \mathrm{DE}$ : media \pm desvío estándar; Me/RIQ: mediana y rango intercuartilo.

PIM2: índice de mortalidad pediátrica; TCS: tejido celular subcutáneo; NAR: neumonía asociada a respirador;

CVC: catéter venoso central; SAMR: Staphylococcus aureus meticilino-resistente; ARM: asistencia respiratoria mecánica;

VAFO: ventilación oscilatoria de alta frecuencia; SDRA: síndrome de dificultad respiratoria aguda;

PELOD: puntaje de disfunción multiorgánica (Pediatric Logistic Organ Dysfunction).

TABLA 2. Tipificación y origen de las infecciones por Staphylococcus aureus según el hospital

\begin{tabular}{|c|c|c|c|c|c|}
\hline Hospitales & SAMR-AC & SAMS-AC & SAMR-N & SAMS-N & Totales \\
\hline Pedro de Elizalde & 15 & 0 & 1 & 2 & 18 \\
\hline Niños de San Justo & 14 & 1 & 0 & 0 & 15 \\
\hline \multicolumn{6}{|l|}{ Niños de } \\
\hline \multicolumn{6}{|l|}{ La Santísima } \\
\hline Trinidad & 2 & 1 & 3 & 4 & 10 \\
\hline Totales & 31 & 2 & 4 & 6 & 43 \\
\hline
\end{tabular}

SA: Staphylococcus aureus; MR: meticilino-resistente; MS: meticilino-sensible; AC: adquirido en la comunidad; N: nosocomial.

TABLA 3. Procedimientos quirúrgicos: 33 actos en 25 pacientes

\begin{tabular}{lc}
\hline Procedimientos & Pacientes \\
\hline Drenaje pleural & 14 \\
Toilette pleural & 6 \\
Decorticación & 2 \\
Drenaje osteoarticular & 4 \\
Desbridamiento muscular & 5 \\
Drenaje pericárdico & 1 \\
Cierre de fístula bronquio-pleural & 1 \\
\hline
\end{tabular}

las UTIP fue la claudicación respiratoria, y se evidenció como principal foco primario el compromiso pulmonar (51\%); se documentó bacteriemia en el 55,8\% de los casos (24 pacientes).

Respecto al antibiograma, $81,4 \%$ de los pacientes (35) tuvo SAMR, con una concentración inhibitoria mínima (solo realizada en el HNPE) de 1,17 . Se evidenció un retraso de 20 h en el tratamiento antibiótico adecuado según antibiograma (10 pacientes).

En 10 pacientes $(23 \%)$, se constató localización metastásica.

En la evolución, 86\% (37) de los ingresos requirieron ARM invasiva; de estos, 26 pacientes $(70 \%)$ desarrollaron SDRA y 9 requirieron VAFO. Se objetivó shock séptico en 27 pacientes (63\%) y el máximo PELOD fue 18,64 $\pm 28,3$.

Se efectuaron 33 actos quirúrgicos en 25 pacientes (Tabla 3). La duración de la estadía en la UTI fue de 13 (5-25) días, y la mortalidad global por infección por SA fue del 14\% (Tablas 1, 2 y 3).

El síndrome clínico definido como NMN-N estuvo presente en el $51 \%$ de los ingresos (20 por SAMR-AC y 2 por SAMS-AC). Las principales características de este síndrome estuvieron presentes en la siguiente proporción: escape aéreo, 54,5\% (12 pacientes); empiema, 50\% (11 pacientes); sangrado de vías aéreas, $41 \%$ (9 pacientes); leucopenia, 36,6\% (8 pacientes); pródromo gripal, 32\% (7 pacientes), y coinfección viral 13,6\% (3 pacientes). 
TABLA 4. Comparación: grupo neumonía necrotizante vs. grupo no-neumonía necrotizante

\begin{tabular}{lccc}
\hline Variables & NMN necrotizante & No NMN-necrotizante & P (<0,05) \\
\hline $\mathrm{N}^{\mathrm{O}}$ & 22 & 21 & 0,970 \\
Edad (meses) & $50,68 \pm 52,90$ & $51,33 \pm 61,34$ & 0,954 \\
Peso (kg) & $18,82 \pm 14,08$ & $18,55 \pm 16,59$ & 0,044 \\
PIM 2 (\%) & $10,36 \pm 11,47$ & $4,42 \pm 6,38$ & 0,698 \\
ARM (días) & 19 & 18 & 0,022 \\
Días libres de ARM a los 28 días & $10,82+10,20$ & $19,09 \pm 9,41$ & 0,002 \\
SDRA & 18 & 8 & 0,001 \\
VAFO & 9 & 0 & 0,015 \\
Adrenalina $>$ 0,25 mcg/kg/min & 14 & $6,19 \pm 21,3$ & 0,031 \\
Máximo PELOD & $26,80 \pm 32,02$ & 10 & 0,223 \\
Drenaje quirúrgico & 15 & $11,1 \pm 9,21$ & 0,024 \\
Estadía en la UTIP (días) & $20,23 \pm 13,38$ & 2 & 0,623 \\
Mortalidad & 4 & 2 & \\
\hline
\end{tabular}

NMN: neumonía; PIM 2: índice de mortalidad pediátrica; ARM: asistencia respiratoria mecánica;

SDRA: síndrome de distrés respiratorio; VAFO: ventilación oscilatoria de alta frecuencia;

PELOD: puntaje de disfunción multiorgánica (Pediatric Logistic Organ Dysfunction).

A fin de objetivar la peor evolución de los pacientes que desarrollaron NMN-N, se compararon los grupos de aquellos que presentaron dicho síndrome con el grupo que no lo presentó (Tabla 4).

El análisis mostró que los pacientes pertenecientes al grupo que desarrolló NMN-N presentaban peores condiciones clínicas al momento del ingreso (PIM2) y peor evolución, evidenciadas por menor cantidad de días libres de ARM a los 28 días, mayor proporción de SDRA y de requerimiento de VAFO, mayor bajo gasto cardíaco, mayor compromiso multiorgánico (PELOD) y estadía más prolongada en UTIP. Estas diferencias fueron significativas estadísticamente. La potencia de nuestra muestra para detectar estas diferencias, considerando un nivel de dos colas, fue del $77 \%$.

\section{DISCUSIÓN}

El SAMR ha sido identificado como un problema mayor de salud pública. En nuestro país, los trabajos de Paganini ${ }^{2-4}$ evidencian una creciente tasa de infecciones por SAMR-AC.

No existen reportes en la literatura local sobre la evolución de las infecciones graves por SA en las UTIP, y son pocos en la bibliografía internacional. ${ }^{12-14}$ Es aquí donde radica la importancia del presente trabajo y la particularidad de incluir tres centros localizados en distintas áreas geográficas.

En nuestro trabajo, el 76,7\% de los pacientes presentó infección adquirida en la comunidad, cifra similar a la reportada por un estudio multicéntrico realizado en nuestro país. ${ }^{3} \mathrm{~A}$ diferencia del citado estudio, la proporción de SAMS-AC fue del $6 \%$.

El principal motivo de ingreso a la UTIP fue la claudicación respiratoria, y el foco primario fue el pulmonar; esta particularidad la comparten los estudios de Cabeza et ál. ${ }^{12}$ y Miles et ál. ${ }^{14}$ En nuestra serie, un alto porcentaje de pacientes requirieron soporte ventilatorio invasivo (86\%) y soporte hemodinámico; trabajos efectuados en otras UTIP ${ }^{12-14}$ reportan cifras similares.

Los valores de mortalidad en las UTIP difieren en la bibliografía: en el trabajo de $\mathrm{Creel}^{13}$ son del $27 \%$, mientras que en nuestra serie son del $14 \%$.

Cabe resaltar que, en las nombradas publicaciones de Cabeza y Miles, la tasa de infección por SAMR fue del $12 \%$ y del $14 \%$, respectivamente, y en nuestro trabajo fue del $81,4 \%$.

Respecto a la NMN-N, la mortalidad fue del $18 \%$, muy por debajo del $56 \%$ que reporta la serie de Gillet. ${ }^{15}$

La principal limitación de este trabajo es su escasa casuística, lo que determina la imposibilidad de realizar un análisis multivariado. Igual- 
mente, no es posible generalizar los resultados obtenidos debido a que se trató de una muestra de conveniencia.

Consideramos, dado el aumento de la incidencia de las infecciones por SA-AC y la tórpida evolución de la NMN-N, que el médico que trabaja en área crítica debe tener un alto índice de sospecha, orientado por guía de directrices, a fin de actuar en forma rápida y eficaz.

\section{CONCLUSIÓN}

Se identificó una alta proporción de infección adquirida en la comunidad; un alto porcentaje de ingresos requirieron soporte ventilatorio y hemodinámico. Se asoció a la NMN-N con peor evolución.

\section{BIBLIOGRAFÍA}

1. Lowy E. Staphylococcus aureus infections. $N$ Engl J Med 1998;339:520-32.

2. Paganini H, Verdaguer V, Rodríguez A, Della Latta P, et al. Infecciones causadas por Staphylococcus aureus resistentes ala meticilina en niños provenientes de la comunidad en niños de la Argentina. Arch Argent Pediatr 2006;104(4):295-300.

3. Paganini H, Della Latta P, Muller Opet B. Estudio multicéntrico sobre las infecciones pediátricas por Staphylococcus aureus meticilino-resistente provenientes de la comunidad en la Argentina. Arch Argent Pediatr 2008;106(5):397-403.

4. Paganini H, Della Latta P, Soto A, Casimir L. Bacteriemias por Staphylococcus aureus adquiridas en la comunidad: 17 años de experiencia en niños de la Argentina. Arch Argent Pediatr 2010;108(4):311-7.

5. Gillet $Y$, Issartel B, Vanhems $P$, et al. Association between Staphylococcus aureus strains carrying gene for Panton-
Valentine leukocidin and highly lethal necrotising pneumonia in young immunocompetent patients. Lancet 2002;359:753-9.

6. Karampela I, Poulakou G, Dimopoulos G. Community acquired methicillin resistant Staphylococcus aureus pneumonia: an update for the emergency and intensive care physician. Minerva Anestesiol 2012;78:930-40.

7. GonzálezB,HultenK,DishopM.PulmonaryManifestationsin Children with Invasive Community-Acquired Staphylococcus aureus Infection. Clin Infect Dis 2005;41:583-90.

8. Kaplan S, Hulten K, González B, et al. Three-year surveillance of community-acquired Staphylococcus aureus infections in children. Clin Infect Dis 2005;40:1785-91.

9. Vos F, Kullberg B, Sturm P, Krabbe P, et al. Metastatic infectious disease and clinical outcome in Staphylococcus aureus and Streptococcus species bacteremia. Medicine 2012;91:86-94.

10. Acute Respiratory Distress Syndrome the Berlin Definition. JAMA 2012;307(23):doi:10.1001/jama.2012.5669.

11. Goldstein B, Giroir B, Randolph A and the Members of the International Consensus Conference on Pediatric Sepsis. International pediatric sepsis consensus conference: Definitions for sepsis and organ dysfunction in pediatrics. Pediatr Crit Care Med 2005;6:2-8.

12. Cabeza B, García Ruiz S, González-Abad M, Nieto-Moroa M. Infecciones por Staphylococcus aureus en cuidados intensivos: características clínicas y epidemiológicas. An Pediatr (Barc) 2012;77:403-12.

13. Creel A, Durham S, Benner K, Alten J, et al. Severe invasive community-associated methicillin-resistant Staphylococcus aureus infections in previously healthy children. Pediatr Crit Care Med 2009;10:323-7.

14. Miles F, Voss L, Segedin E, Anderson B. Review of Staphylococcus aureus infections requiring admission to a paediatric intensive care unit. Arch Dis Child 2005;90:12748.

15. Gillet $Y$, Vanhems P, Lina G. Factors Predicting Mortality in Necrotizing Community-Acquired Pneumonia Caused by Staphylococcus aureus Containing Panton-Valentine Leukocidin. Clin Infect Dis 2007;45:315-21. 